\title{
Role of cerebral angiography in vertebrobasilar occlusive disease
}

\author{
LOUIS R. CAPLAN AND ARTHUR E. ROSENBAUM \\ From the Departments of Neurology and Radiology, Harvard Medical School, \\ The Beth Israel Hospital, and the Peter Bent Brigham Hospital, Boston, Massachusetts, U.S.A.
}

SYNOPSIS The authors attempt to separate clinical subgroups of patients within the larger category of vertebrobasilar artery disease, and to indicate the present role of angiography in their recognition and management. Angiography is of use in separating posterior fossa occlusive vascular lesions from space occupying lesions. In addition, by defining the locus and nature of the occlusive process, it may result in more rational treatment and prognostication. Subgroups of vertebrobasilar ischaemia which have a favourable prognosis may be separable clinically or, in unclear cases, angiographically.

Recognition and description of clinical syndromes of brain-stem infarction flourished in the late 19th and early 20th centuries (Weber, 1863; Jackson, 1864; Raymond and Cestan, 1901; Wallenberg, 1901). It was not until 1946, however, that Kubik and Adams described occlusion of the basilar artery in postmortem examinations. These authors emphasized a dire prognosis. Subsequent reports described the clinical picture, which was called vertebrobasilar insufficiency (Denny-Brown, 1953; Siekert and Millikan, 1955; Fang and Palmer, 1956; Williams and Wilson, 1962; Williams, 1964); Browne and Poskanzer (1969), in a review of stroke treatment, also emphasized the grave nature of the disease: 'If anticoagulation has value, it may be more useful in the patient with vertebrobasilar disease, with its high mortality, than in other forms of cerebral vascular thrombosis'.

Marshall's (1964) review of the natural history of transient ischaemic attacks uncovered a relatively better prognosis for patients with vertebrobasilar ischaemic episodes than for those patients with carotid ischaemia. Furthermore, several groups of patients with ischaemic disease of the posterior cerebral circulation seem to have a relatively good prognosis. Fisher described several syndromes produced by lacunar

Address for correspondence: Dr Louis R. Caplan, Department of Neurology, Beth Israel Hospital, Boston, Massachusetts 02215, U.S.A.

(Accepted 10 February 1975.) infarction in the brain-stem related to small vessel disease associated with hypertension: the dysarthria-clumsy hand syndrome (Fisher, 1967), pure motor hemiplegia (Fisher and Curry, 1965), pure sensory stroke (Fisher, 1965b), and homolateral ataxia and crural paresis syndrome (Fisher and Cole, 1965). These syndromes have a favourable outlook for recovery. Despite numerous episodes of vertebral insufficiency, not a single case of pathologically documented brain-stem infarction was found in a review of over 100 cases of subclavian steal syndrome (North et al., 1962; Patel and Toole, 1965; Wheeler, 1967; Baker et al., 1973). The pure syndromes of lateral medullary or lateral pontine infarction also have a good prognosis (Currier et al., 1958). Fisher (1970) has pointed out that occlusion of the vertebral artery in the neck commonly produces transient symptoms but rarely produces brain-stem infarction unless the vertebral artery is occluded intracranially. Thus it must be clear that vertebrobasilar disease is not a homogeneous entity; some clinical subgroups have relatively better prognoses than others.

The outcome in any given patient with posterior circulation occlusive disease is dependent on: (1) the particular anatomy of the vertebrobasilar circulation (developmental variations are very common); (2) the locus and rapidity of the occlusive process; (3) the physiological state of 

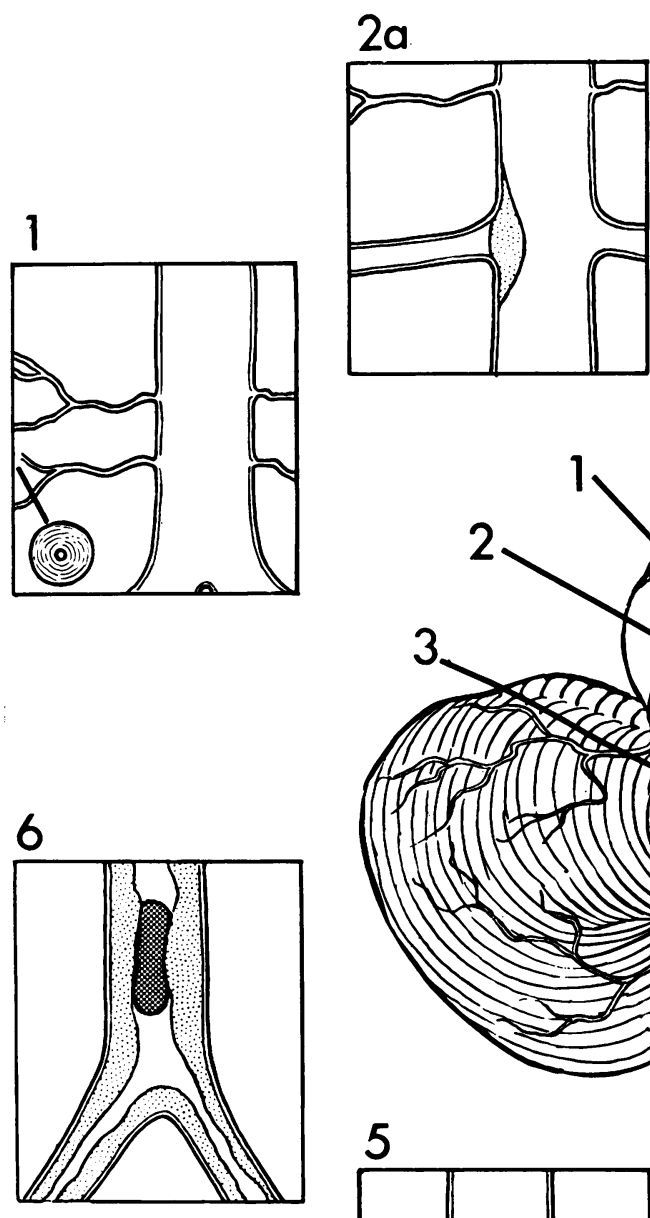

$2 b$
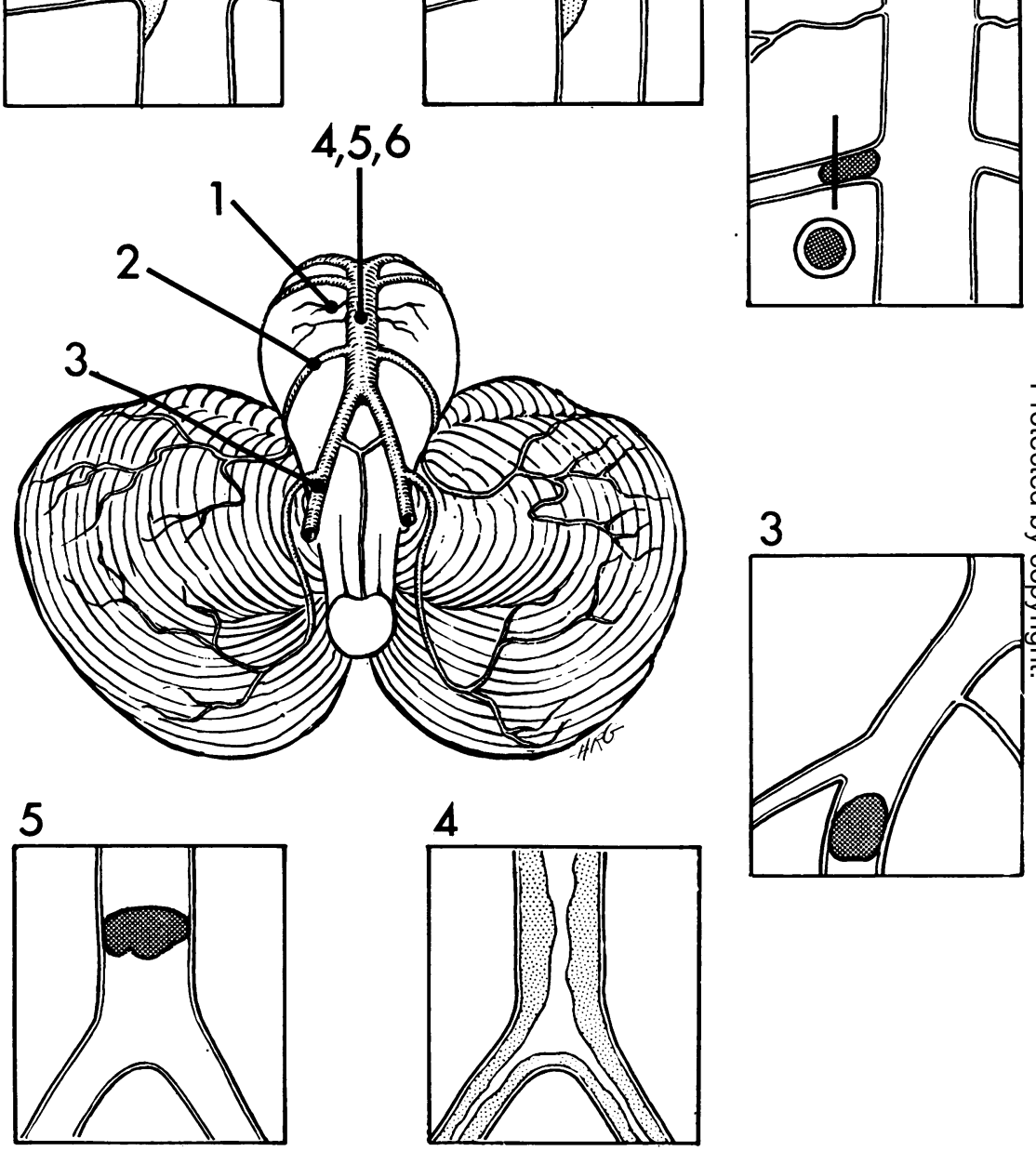

FIG. 1 Major forms of vascular occlusion in the posterior circulation. 1. Medial hypertrophy of small arteries. 2. Obstruction of major vessel branches: a. atheroma in lumen of parent vessel blocking orifice; b. atheroma extending into branch; c. thrombotic occlusion of branch. 3. Occlusion of vessel proximal to branch origin. 4. Stenosis of large vessel by atheroma. 5. Embolic occlusion of vessel. 6. In situ thrombosis complicating atheromatous stenosis of a large vessel. 
the systemic circulation and haematological system; and (4) the availability of adequate collateral circulation; congenitally deficient or previously obstructed cranial and extracranial vessels may diminish available collateral vessels.

Six major types of vascular pathology may be seen in occlusive disease of the posterior cerebral circulation (Fig. 1): (1) disruption of small penetrating branches of vessels as seen in hypertension or arteritis (Fisher, 1969); (2) obstruction of branch vessels produced by atheroma blocking the origin of the branches or by intrinsic atheroma or thrombosis of the larger branches themselves; (3) occlusion of a vessel proximal to the origin of the branch (Fisher et al., 1961); (4) major stenosis of the intracranial vertebral arteries bilaterally or of the basilar artery; (5) thromboembolic occlusion of major cerebral vessels arising from the heart, aortic arch, or vertebral arteries; (6) in situ thrombosis of the vertebrobasilar arteries themselves. It seems likely that the last three pathological entities would be associated with more serious infarction and a more grave clinical outcome. Also these varying pathologies would probably respond quite differently to therapeutic endeavours such as anticoagulation. Castaigne et al. (1973) have reviewed the loci and nature of vascular changes in patients with occlusions in the vertebrobasilar system discovered during postmortem examinations.

The clinical differentiation between small and large vessel disease is frequently difficult. Occlusions of small, penetrating branch-vessels generally occur in hypertensive patients. The anatomy of the infarct is usually limited to territories supplied by a single median, paramedian, or circumferential vessel. Small vessel lesions are usually not accompanied by prominent headache, and the duration of time between original ischaemic symptoms and completed stroke is usually short (hours to weeks). In contrast, large vessel disease-for example, basilar artery-often produces bilateral signs not produced by occlusion of a single penetrating branch: the symptomatic period may be prolonged, the symptoms more variegated and less stereotyped, and headache may be prominent. Branch occlusion may be attended by more widespread signs, however, depending on the integrity of other branches and the general state of the circulation.

With the frequent use of selective vertebral angiography and magnification radiography, it is now possible to define the individual anatomy and vascular pathology in many cases of posterior circulation occlusive disease. By defining the vascular lesion, angiography may help the clinician not only to prognosticate but to select the most appropriate treatment. Angiography also may help separate occlusive vascular disease from haemorrhage or tumour of the posterior fossa. In this paper, we analyse 11 recent cases of occlusive vascular disease of the posterior circulation studied clinically and angiographically to illustrate the role of angiography in clinical management of vertebrobasilar disease.

\section{CASE 1}

S.M., a 55 year old insulin-dependent, diabetic gypsy man had a 14 year history of hypertension and three previous myocardial infarctions. A stroke had produced left hemiparesis which cleared after a few months. One day before admission, he awakened with dysarthria and right facial weakness which resolved in 30 minutes. The next day dizziness, right facial weakness, and dysarthria recurred but fluctuated during the day until right hemiplegia developed. Examination revealed complete right hemiplegia and shivering of his left limbs. The next day he became quadriplegic and developed bifacial paresis, dysphagia, and severe dysarthria. Electroencephalography was normal. Transfemoral cerebral angiography (Figs $2 a-c$ ) demonstrated no filling of the basilar artery above the level of the anterior inferior cerebellar arteries. The left posterior inferior cerebellar artery filled the left superior cerebellar artery resulting in retrograde opacification of the upper basilar artery. Bilateral carotid angiography showed no supratentorial circulatory abnormality. He was placed on anticoagulation therapy and was able to walk with help 11 months later. No additional ischaemic episodes have occurred in the 33 months since the original stroke.

COMMENT The presence of hypertension and diabetes suggested either small or large vessel disease in this patient. The clinical picture, however, was more compatible with large vessel occlusion of the basilar artery. He was a suboptimal candidate for anticoagulation because 


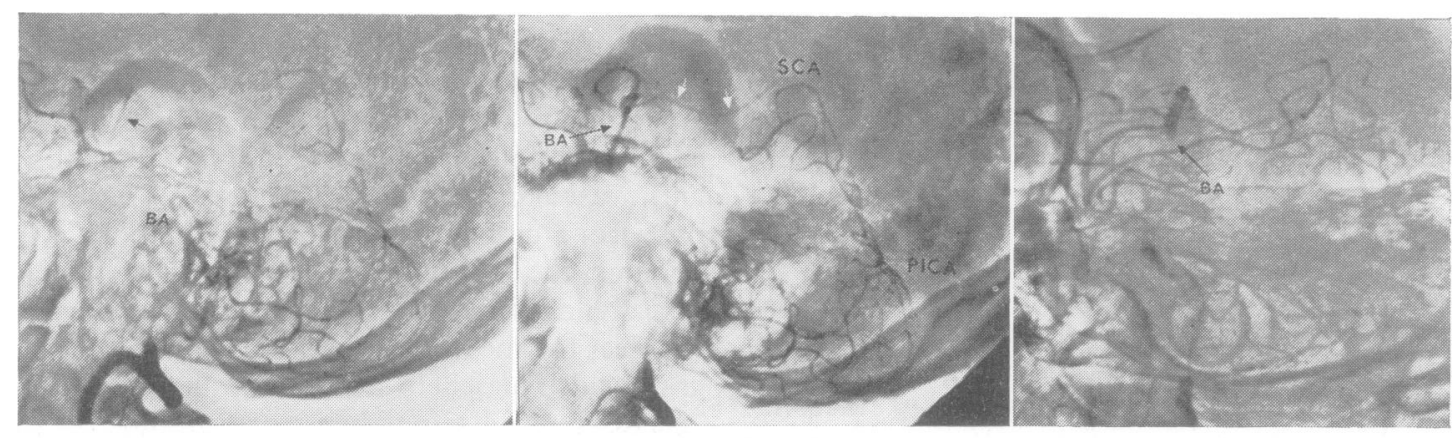

(a)

(b)

(c)

FIG. 2 Case 1. Near total occlusion of the basilar artery between the superior cerebellar and anterior inferior is cerebellar branchings. Collateral flow from posterior inferior cerebellar artery (PICA) to superior cerebellar $\vec{\circ}$ artery (SCA). (a) Lateral projection: transient filling of the rostral basilar artery (arrow) is via the stenotic $\rightarrow$ segment. (Distal basilar artery, BA.) (b) Lateral projection; two seconds later: greater filling of the superior $\vec{\omega}$ cerebellar artery has resulted from flow through SCA branches (superior vermian and marginal). The posterior cerebral artery is faintly filled (white arrows). (c) $45^{\circ}$ cross-table oblique projection: the central ray courses from laterally between the petrous pyramids and shows the superior limit of the basilar artery (BA) occlusion just $\dot{\omega}$ below the origin of the superior cerebellar arteries.

of his hypertension and nomadic nature. As there was arteriographic confirmation of basilar occlusion, anticoagulant therapy was used despite relative contraindications.

\section{CASE 2}

D.S., a 78 year old mildly hypertensive widow, gave a six month history of intermittent dizziness with staggering. On one occasion she recognized transient dysarthria associated with faintness. Six days before admission she noted weakness of her legs on rising. Later the same day her left face, arm, and leg were weak. The weakness gradually increased, and on the morning of admission there was diplopia on right gaze and paraesthesiae of the left hand. Examination revealed a partial right sixth nerve palsy, a moderate left hemiparesis, diminished palatal motion, dysarthria, and bilateral extensor plantar reflexes. The right sixth nerve palsy gradually cleared. Selective left vertebral angiography demonstrated normal vertebral arteries and a minimally irregular basilar artery without stenosis or occlusion. She made an excellent recovery clinically and remains selfsufficient at home two years later without further treatment.

COMMENT The clinical findings fit best with a basilar artery branch occlusion producing a right median pontine infarction. The reduced palatal movement, extensor plantar reflexes, bi $c$ lateral leg weakness, and six month history of transient ischaemia with dizziness did raise the $\overrightarrow{0}$ question of disease of a larger vessel. Arterioz or graphy failed to reveal serious large vessel pathology and led to the decision not to use anticoagulants.

\section{CASE 3}

A.W., a 46 year old mildly hypertensive Chinese man had had a myocardial infarction a year earlier. During the two weeks before admission, he had three transient episodes of nausea, vertigo, and ataxia. Examination revealed a right sixth nerve palsy and a tendency to fall to the right while erect. In the hospital, he developed left hemianopsia. Right vertebral angiography filled the posterior inferior cerebellar arteries bilaterally but the basilar artery was only faintly opacified (Fig. 3a). Left internal carotid arteriography (Fig. 3b) showed retrograde filling of the superior portion of the basilar artery with excellent filling of the superior cerebellar vessels. The basilar artery was occluded for a short $N$ segment between the level of its anterior inferior $N$ cerebellar and superior cerebellar artery branches. The patient did well on anticoagulant therapy and has had no recurrent episodes. 


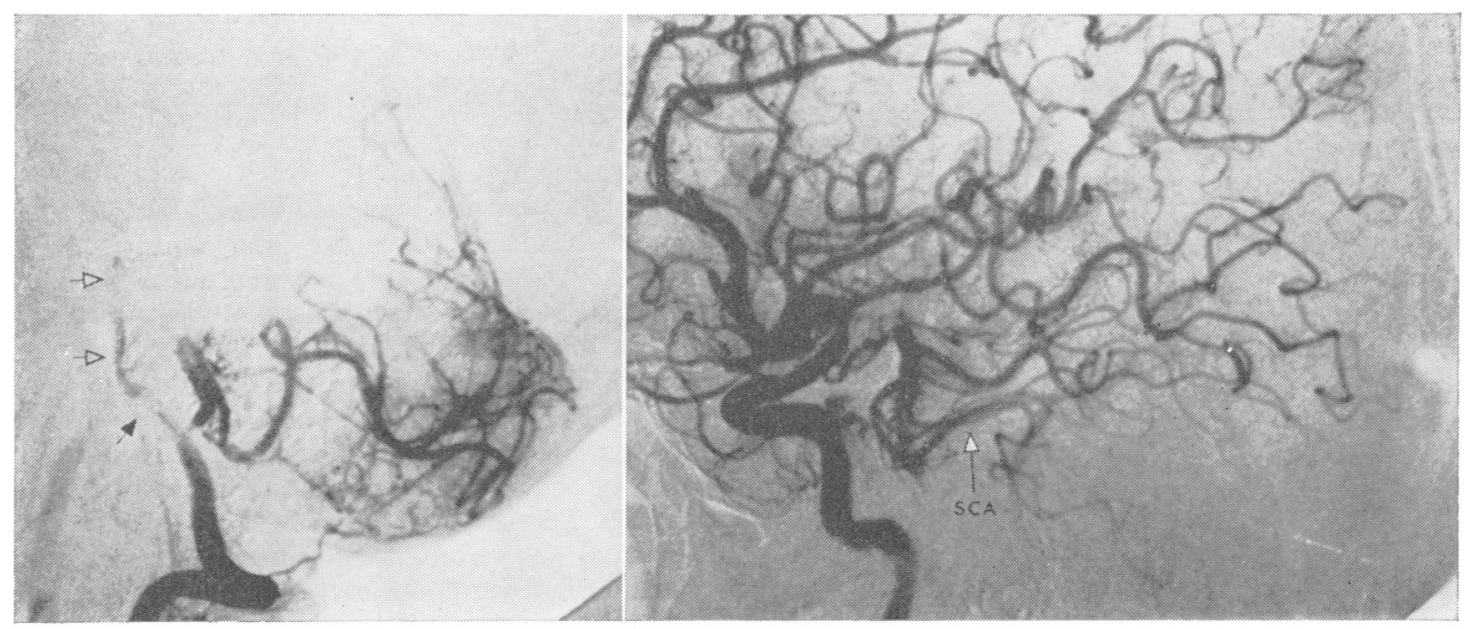

(a)

(b)

FIG. 3 Case 3. Focal occlusion of basilar artery below the origins of the superior cerebellar arteries. (a) Selective right vertebral arteriography-lateral projection: the right posterior inferior cerebellar artery filled well despite the prominent stenosis at its origin. Contralateral posterior inferior cerebellar branches subsequently fill well. Above the level of the right PICA origin, the basilar artery (solid arrow) diminishes in calibre and washout from this segment is slow. Only the posterior inferior surface of the cerebellum opacifies. (b) Left internal carotid angiography -lateral projection: via a small posterior communicating artery, the ipsilateral posterior cerebral and both superior cerebellar branches (SCA) fill well; the contralateral posterior cerebral artery is opacified, but less densely. The segment of occlusion between the proximal (Fig. 3b) and distal (Fig. 3a) portions of the basilar artery is short.

COMMENT This patient's clinical picture of cerebellar signs and symptoms along with a right sixth nerve palsy and right occipital lobe lesion was confusing. Angiography demonstrated the serious nature of the vascular process, a clinically unsuspected basilar occlusion. These findings led to the choice of anticoagulant therapy.

\section{CASE 4}

F.B., a 77 year old female with peptic ulcer disease, had a three week history of dizziness with falling and frontal headache. Two days before admission she noticed episodic dizziness, dysarthria, and inability to stand. On the day of admission, there were six episodes of dysarthria and quadriparesis. Examination during a spell revealed severe dysarthria, bifacial paresis, tongue protrusion to the left, quadriparesis greatest in the left limbs, and bilateral extensor plantar reflexes. Between spells only a mild left facial weakness and slight left ataxia were seen. Countercurrent right brachial arteriography showed severe irregularity and stenosis in the superior extreme of the right vertebral artery and the adjacent inferior portion of the basilar artery. She was treated with anticoagulants and had not had a subsequent stroke in two years of follow up. When the anticoagulant was stopped temporarily a year after admission, she again became vertiginous and fell on several occasions. These symptoms ceased upon reinstitution of the therapy.

COMMENT This elderly woman had a relative contraindication to anticoagulation (peptic ulcer disease) with intermittent but severe symptoms of bilateral brain-stem dysfunction. Angiography corroborated the serious nature of the underlying pathology and led to anticoagulation with special attention to ulcer management.

\section{CASE 5}

H.T., a 49 year old man with a gastrectomy for peptic ulcer disease, had no history of hypertension or cardiac disease but complained of intermittent 
pain and coldness in his feet. For two weeks before admission, he had intermittent blurred vision and headache. Ataxia, dizziness, a weak feeling in the legs, dysarthria, perioral paraesthesiae, and a feeling that his eyes were turning were variably present at the time of visual blurring. Examination between attacks was normal. Intravenous heparin therapy was instituted. Vertebral angiography showed normal vertebral and basilar arteries with slow flow and some delayed emptying. A careful haematological survey revealed a haemoglobin level of $16.7 \mathrm{~g} / \mathrm{dl}$, and an elevated platelet count of $360000 / \mathrm{mm}^{3}$ but no definite clotting disorder. Aspirin ( $1 \mathrm{~g}$ per day) led to a remarkable alleviation of his neurological and peripheral vascular symptoms.

COMMENT The clinical history clearly suggested vertebrobasilar ischaemic spells and peripheral vascular disease. The absence of significant vessel disease on cerebral angiography resulted in a careful systemic search. The haematological disorder was uncovered but is still poorly characterized. The remarkable benefit from aspirin was believed to be related to its effect on platelet agglutination.

\section{CASE 6}

S.S., a 60 year old man had complained of intermittent headache, dizziness, and nausea for six months. A month before hospitalization he had a spell of left-sided weakness lasting for several hours. On the day of admission he had felt weak at work and fell. Examination revealed a complete left hemiplegia. Cranial nerves were normal except for persistent burning pain and tingling in his face bilaterally, worse on the right. A glucose tolerance curve showed diabetes. Left vertebral angiography (Fig. 4) revealed a normal left vertebral artery with transient reflux into the right vertebral artery. A few millimetres of the caudal portion of the basilar artery filled (Figs $4 \mathrm{a}$ and $\mathrm{c})$. The superior cerebellar artery filled from hypertrophied hemispheric branches of the posterior inferior cerebellar artery (Fig. 4b), resulting in opacification of the rostral basilar artery. Carotid angiography was normal with no opacification of the

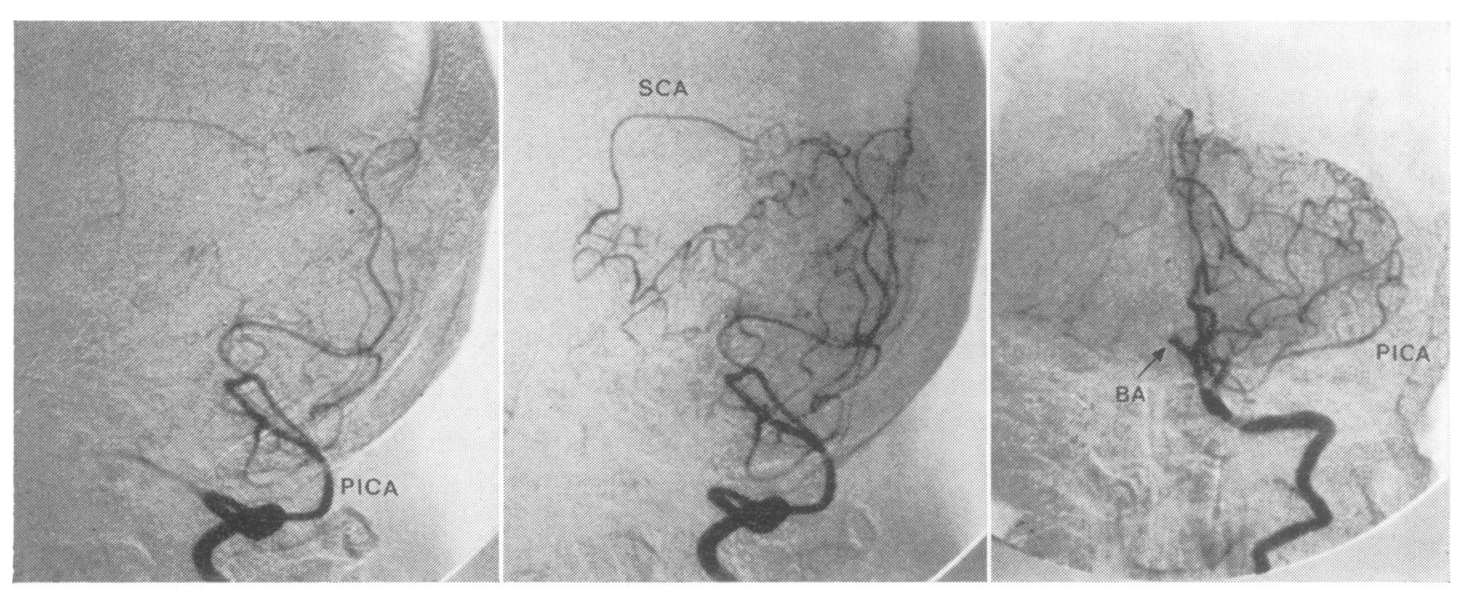

(a)

(b)

(c)

FIG. 4 Case 6. Excellent posterior inferior cerebellar to superior cerebellar collateral circulation in distal basilar artery occlusion. (a) Selective, left vertebral arteriography-lateral projection: the junctional segment between the distal basilar artery and the origin of the posterior inferior cerebellar artery (PICA) fills transiently. The PICA is well developed and, via the inferior vermian branches, rapidly opacifies the superior vermian territory of the superior cerebellar artery. Vertebral artery, VA. (b) One second later: both superior vermian and marginal branches of the superior cerebellar artery (SCA) have opacified without basilar artery filling. (c) Chamberlain-Townes projection: only the distal extreme of basilar artery (BA) is shown on selective vertebral angiography. The hemispheric branches of the posterior inferior cerebellar artery (PICA) are hypertrophied and connect with hemispheric branches of the superior cerebellar artery. The PICA to SCA vermian connections lie in the midline. 
vertebrobasilar system. He has done well on anticoagulants.

COMMENT Clinically, the lesion seemed to involve a penetrating branch of the basilar artery. However, the long duration of symptoms and persistent bilateral facial paraesthesiae raised the question of large vessel disease. The latter diagnosis was confirmed by angiography.

\section{CASE 7}

J.L., a 72 year old man, had complained of recent severe lethargy and vomiting felt to be related to depression. Examination revealed a lethargic man with horizontal nystagmus and left limb ataxia. Subsequently bilateral limb and facial paresis developed with bilateral extensor plantar signs and stupor. An electroencephalogram was diffusely slow. Angiography revealed an obliterated left vertebral artery and severe narrowing of the right vertebral artery with no intracranial basilar artery filling. Heparin was administered, but the patient became comatose and died.

COMMENT The initial clinical impression of metabolic encephalopathy was unlikely because of the lateralized cerebellar signs. Angiography defined a bilateral block of vertebral artery flow and clarified the diagnosis of infarction in the posterior cerebral circulation rather than haemorrhage or mass lesion. The patient succumbed despite the use of heparin.

\section{CASE 8}

M.B., a 58 year old man, suddenly became vertiginous, veered to the left, and felt an odd sensation in his left limbs a month before admission. These symptoms cleared after two days, but two weeks later he noticed pain in his left eye and nose and an odd metallic taste in his mouth as well as left-sided weakness. At another hospital, left sixth nerve palsy, nystagmus, and left hemiparesis were found. Right carotid angiography was normal. He developed stiffness of his legs and transient stupor which improved after heparin administration. Examination revealed a left Horner's syndrome, decreased left corneal reflex, a left sixth nerve palsy, diminished hearing on the left, left ataxia, and bilateral extensor plantar reflexes. The left vertebral artery could not be catheterized from either the left subclavian artery or aortic arch. The thyrocervical trunk was injected and muscle branches of the left vertebral artery opacified with opacification of the near inferior extreme of the left vertebral artery. Right vertebral angiography revealed normal posterior inferior cerebellar arteries and basilar artery with retrograde flow down the left vertebral artery. He has done well on anticoagulant treatment.

COMMENT This patient had two sudden episodes of brain-stem dysfunction, one thalamic and the other lateral pontine. Angiography revealed an occluded inferior extreme of the left vertebral artery in the neck which was the likeliest source of embolization to the posterior circulation intracranially. Anticoagulation was used to prevent subsequent embolization.

\section{CASE 9}

D.T., a 72 year old diabetic hypertensive female, complained of headache and difficulty with walking for several months. Examination was normal except for a shuffling gait, bilateral extensor plantar signs, and inconsistent horizontal nystagmus. A day after admission, lethargy, bifacial weakness, vertical nystagmus, and vomiting were noted. The clinical diagnosis was uncertain, but a space occupying lesion of the posterior fossa was suspected. Angiography revealed arteriosclerosis and delayed washout from the (smaller) right vertebral artery (Fig. 5a). Blood flow through the (larger) left vertebral artery was greatly diminished (Fig. 5b). An occlusion of the midportion of the basilar artery was present. Both posterior cerebral and superior cerebellar arteries filled via leptomeningeal anastomoses after middle cerebral artery opacification (Fig. 5c-d). Subsequently she developed bilateral decorticate posturing and died despite anticoagulation therapy. Postmortem examination revealed an old, partially recanalized occlusion of the basilar and right vertebral arteries. Extensive recent infarction affected the cerebral peduncles, pons, cerebellum, and medial occipital lobes. The mid-pons was almost totally necrotic.

COMMENT A confusing clinical picture of headache and shuffling gait suggested a mass lesion. Angiography demonstrated bilateral slowing of vertebral blood flow and mid-basilar artery occlusion and clarified the diagnosis. She died despite anticoagulation.

\section{CASE 10}

D.V., a 62 year old hypertensive diabetic man, after a day of chills and malaise, suddenly became un- 


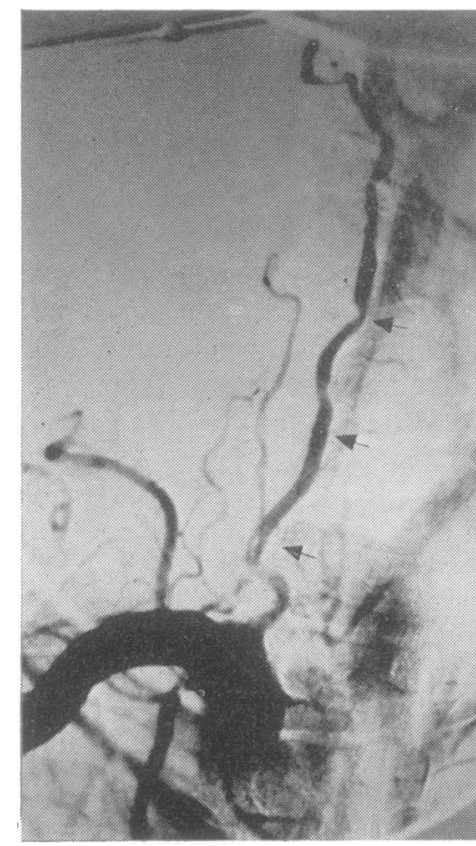

(a)

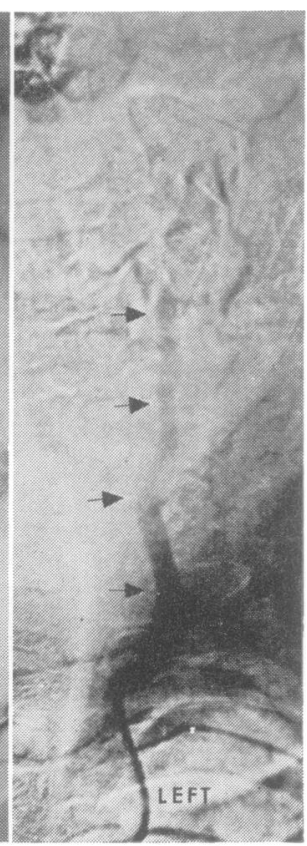

(b)
FIG. 5 Case 9. Acute caudal basilar artery occlusion with thrombosis involving vertebral arteries. (a, top left) Right subclavian arteriographyfrontal oblique projection: although the subclavian artery is densely opacified, flow through the right vertebral artery (arrows) is slowed. The vertebral artery is tortuous and irregular in calibre and less densely opacified than the subclavian or internal mammary arteries. Washout from it is slow relative to that of other cervical and subclavian branches. (b, top right) Left vertebral arteriography -frontal oblique projection: at the time of injection, considerable reflux of the $6 \mathrm{ml}$ bolus occurred into the subclavian artery but disappeared quickly. This angiogram (obtained seven seconds after injection) shows filling of only the proximal portion of this vertebral artery (arrows). (c, left) Left carotid angiography-lateral projection, midarterial phase: diffuse advanced changes of arteriosclerosis affect the anterior and middle cerebral branches. Posterior temporal branches (MCA) of the middle cerebral artery are well filled peripherally. (d, right) Left carotid angiography-late arterial phase. Retrograde filling of posterior $\vec{\Phi}$. cerebral branches peripherally (PCA, white $\stackrel{\circ}{\Phi}$ 을 arrows) from the middle cerebral artery via leptomeningeal anastomosis results in filling of the superior portion of the basilar artery with inferior $ᄋ$ flow into the superior cerebellar branches (SCA).

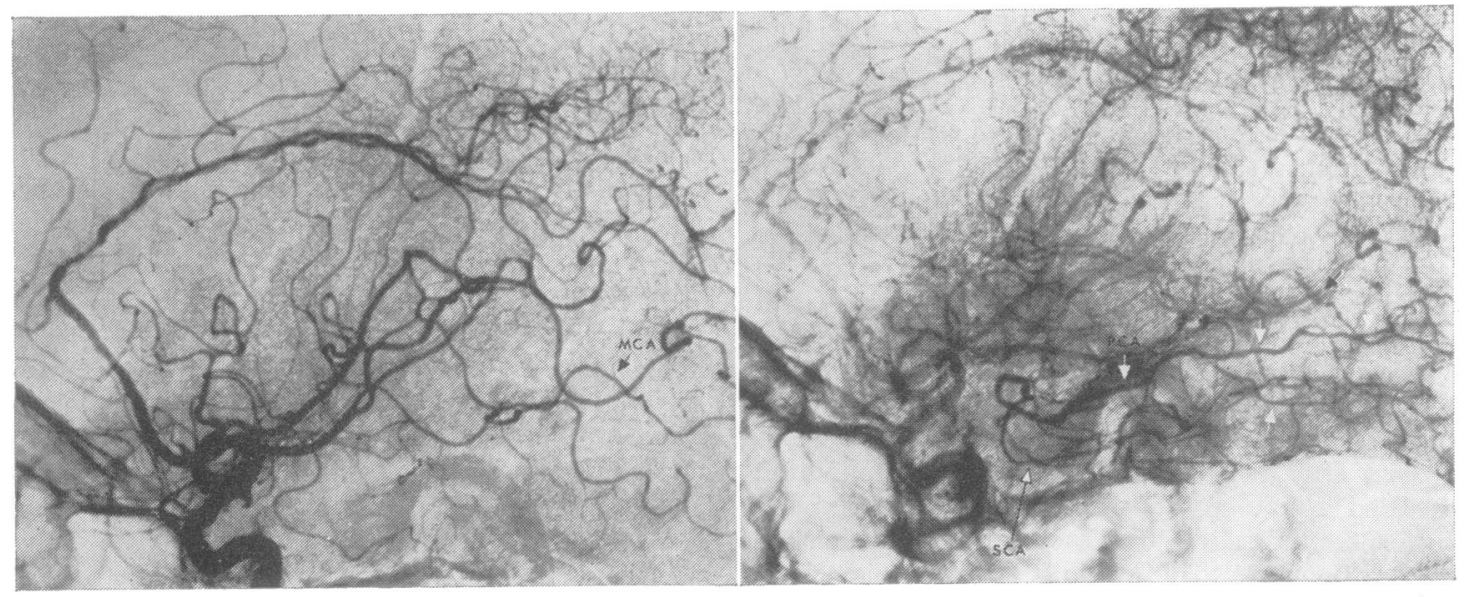

(c)

(d) 


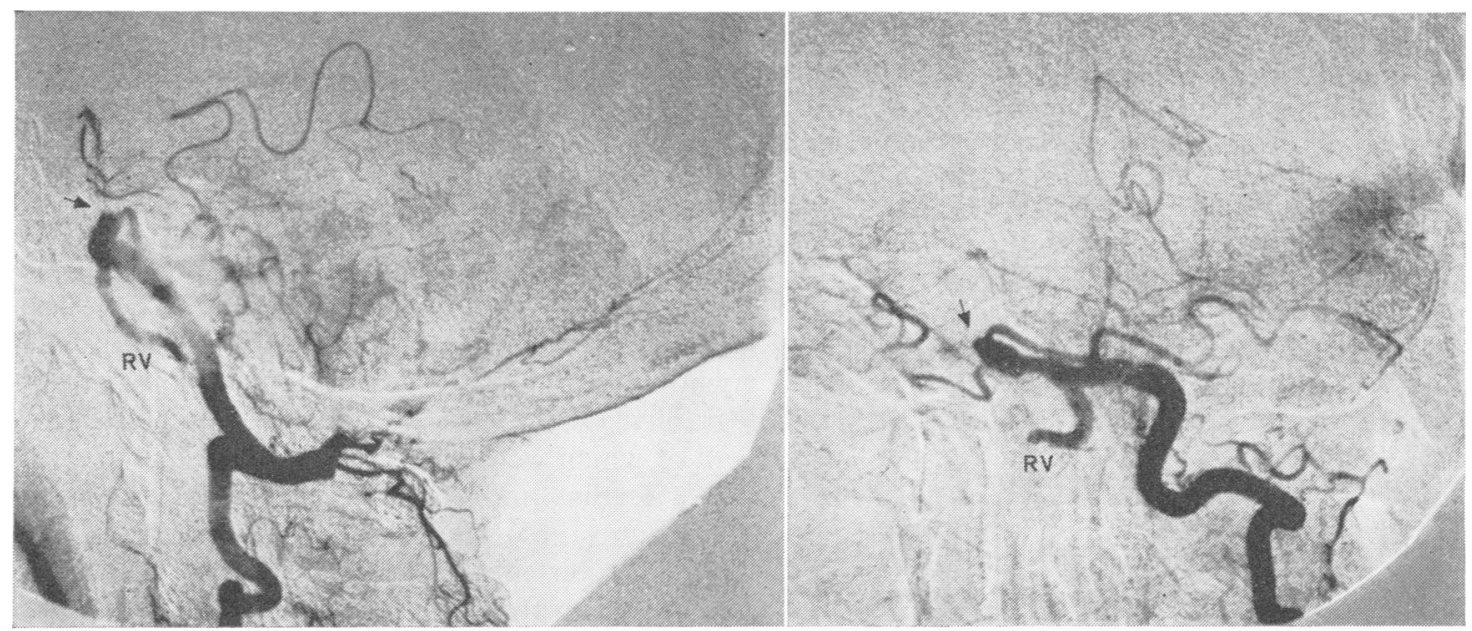

(a)

(b)

FIG. 6 Case 11. Collateral circulation via large anterior inferior cerebellar arteries in inferior basilar artery occlusion. (a) Left vertebral arteriography-lateral projection, early arterial phase. Excellent opacification of the left vertebral artery results in reflux into the right vertebral artery (RV) with demonstration of an irregularly marginated basilar obstruction just beyond the origin of the large AICAs. No posterior inferior cerebellar branches were visualized. (b) Frontal projection-arterial phase similar to (a). Hemispheric branches communicating with the superior cerebellar territory are visualized. The occlusion of the basilar artery is again seen (arrow). Right vertebral, RV.

responsive while defaecating. Pneumococcal pneumonia with high fever was recognized and treated with antibiotics. On the fourth day of illness, he was alert but memory function was poor. When asked to describe a picture, the patient would omit parts of the picture and would have difficulty in comparing sizes and distances within the picture. He would also omit words and sentences within a paragraph. Bilateral extensor plantar reflexes were present as well as a minimal left arm weakness. After initial improvement, a left gaze palsy and somnolence developed. Electroencephalography revealed diffuse delta slowing. On the eighth hospital day, he became deeply comatose. Examination revealed small reactive pupils, no reflex eye movements, and bilateral decerebrate posturing. Left vertebral angiography revealed delayed flow with no opacification above the left posterior inferior cerebellar artery. By analysing the plain radiographs of the neck and comparing foramina transversaria, it was clear that the left vertebral artery was dominant. Basilar occlusion was suggested. He died despite rapid heparinization. Postmortem examination revealed occlusion of the right vertebral artery and lower basilar artery in an area of considerable atheroma. There was extensive infarction involving the midbrain, pons, thalami, and bilateral temporal and occipital lobes.

COMMENT The early clinical picture was puzzling because of the prominence of hemispheral signs (diminished alertness with poor visual and memory function) and the absence of clinical signs of brain-stem dysfunction. Fever and sepsis were complicating issues. Metabolic encephalopathy, infection of the central nervous system, or infarction of watershed zones of the brain were early possible diagnoses. Angiography clarified the diagnosis of occlusive disease of the posterior cerebral circulation. Careful scrutiny of plain radiographs of the neck aided in confirming that delayed emptying involved the dominant vertebral artery.

\section{CASE 11}

V.I., a 58 year old hypertensive man, had suffered intermittent spells of dizziness, diplopia, and generalized weakness for one week. On the day of admission he awakened with dizziness. A right hemi- 
paresis and later transient apnoea occurred while he was being examined in the Emergency Ward. Examination revealed bilateral partial sixth nerve palsies and a slight right hemiparesis. Heparin therapy was instituted. While he was in hospital, the symptoms fluctuated considerably and were worse when he sat or stood. Left vertebral angiography revealed occlusion of the basilar artery just distal to the bilaterally large anterior-inferior cerebellar artery branches (Fig. 6a-b). The posteriorinferior cerebellar arteries were not visualized. Anterior-inferior cerebellar artery branches filled the superior cerebellar artery and distal basilar artery. He was placed on anticoagulants and had no symptoms 15 months later.

COMMENT The clinical picture of bilateral abducens palsies and fluctuating weakness indicated vertebrobasilar artery disease. Because of the possibility of basilar aneurysm and hypertension, which represents a relative contraindication to long-term anticoagulation, angiography was performed. The presence of a total basilar occlusion led to anticoagulation.

\section{DISCUSSION}

Meyer et al. (1960) published their findings on 35 patients in whom angiography of the posterior intracranial circulation was performed by percutaneous subclavian or vertebral injection or by retrograde injection into the carotid artery. A high incidence of stenosis of the basilar artery (seven out of 35) and stenosis of the cervical vertebral artery were found. They commented:

'In the majority of patients with insufficiency of the vertebral basilar system, the main site of disease is in the basilar artery itself, and in those with disease of the cervical portion of the vertebral artery there is often associated atherosclerosis of the basilar artery.'

Since that time there have been several important advances in the clinical-pathological correlation of posterior circulation strokes, and many advances in angiographic technique and capabilities. Fisher (1965a, 1967) has delineated the clinical and pathological picture related to brain-stem lacunae, and Fisher and Caplan (1971) have discussed the pathology of basilar branch occlusions.

The advent of low-toxicity contrast media and advances in the technique and experience of the personnel performing vertebral angiography have made this procedure safer. A review of several large recent series indicates that serious complications are surprisingly rare. In a large Scandinavian series of over 200 cases of vertebral angiography (Törmä and Fogelholm, 1967), seven of 249 patients undergoing vertebral angiography had serious neurological complications all of which cleared within 72 hours. Weibel and Fields (1966) reported on 846 patients who underwent trans-subclavian vertebral angiography. Of these, 9\% developed complications, all of which were local and minimal. There were no deaths and no cerebral complications. Pribram (1965) cites two serious complications in 357 vertebral studies: an asymptomatic occlusion of the vertebral artery and the other a cerebellar syndrome.

The Seldinger technique for selective catheterization of individual brachiocephalic arteries allows safe and more extensive study of the posterior circulation. Radiographic magnification provides greater resolution and better vascular detail and is used routinely in our cases. Radner (1951), Schechter and Zingesser (1965), and Pochaczevsky et al. (1971) have reviewed the radiological anatomical details of posterior circulation angiography and have pointed out some pitfalls in analysis of these studies. Others have commented extensively on the radiographic features of vertebrobasilar occlusive disease. Extensive analysis of the venous anatomy of the posterior fossa has not only corroborated arterial findings but can be equally or more valuable in the diagnosis of space-occupying vascular lesions in the posterior fossa (such as cerebellar haemorrhage or an oedematous cerebellar infarction). Since both cerebellar infarction and cerebellar haemorrhage can produce death by brain-stem compression and are often successfully treated by decompressive surgery (Lehrich et al., 1970), differentiation of them and appropriate surgical planning can be life-saving.

The occlusive nature of the pathology in our patients was identified angiographically in cases 7,9 , and 10 , in whom the suggested clinical diagnoses were a metabolic disturbance or a space-occupying lesion. In other cases, clinically considered to be vascular in nature, angiography was instrumental in defining whether the disturbance involved small or large vessels. 
Several patients with rather minor signs were surprisingly found to have complete basilar occlusions (cases 3 and 6). Two patients with rather ominous clinical complaints and signs proved to have very little large vessel pathology angiographically (cases 2 and 5) and were spared long-term anticoagulation. Four other patients (cases 1, 3, 4, and 11) who were poor anticoagulant risks had the diagnosis of severe basilar obstruction verified and were treated with anticoagulants despite the risks involved. A source of posterior circulation embolization was identified in another patient (case 8) in the form of an occluded vertebral artery. A disturbance in flow without anatomical obstruction was suggested in case 5 and was perhaps related to a haematological disorder. The remarkable response to aspirin is of interest in this case and was considered to be related to its effect on platelet agglutination. Four of our patients with verified basilar artery occlusion are alive at 30 , 29, 33, and 15 months after the stroke (cases 1,3 , $6,11)$. One of the patients has a residual paraparesis and another a mild hemiparesis; the other two patients have only minimal residual signs and are essentially well. It is not well appreciated that patients may indeed survive for a prolonged period after a total basilar occlusion without disabling neurological sequelae. Two of the patients (cases 7 and 9) had bilateral vertebral artery occlusion. In our experience, bilateral occlusion of the intracranial vertebral artery has often presented a confusing clinical picture of ataxia, visual disturbance, and altered mental function which has generally terminated fatally. The diagnosis may not be evident until arteriography of the posterior circulation has been performed.

Unfortunately, optimal therapy of occlusive disease of the posterior circulation remains an unsettled question. Early studies of anticoagulation in vertebral basilar disease did indicate some possible benefit (Millikan et al., 1955, 1958; Hill et al., 1960). However, these early clinical studies grouped brain-stem ischaemia as a single entity and only occasionally considered transient ischaemic attacks, strokes in evolution, and completed strokes as separate subgroups within this larger group. The underlying vascular anatomical and pathological derangements were not considered. Little knowledge of the natural history of the disease was then available for comparison with the treated group. The role of heparin and other anticoagulants needs clarification especially in those patients with serious vascular lesions. The vascular anatomy, and temporal state of the stroke (transient ischaemic attack, stroke in evolution, or completed stroke) should be important factors in deciding the type of therapy instituted. The role of agents which diminish platelet agglutination-for example, aspirin and dipyridamole - in the therapy of vertebral basilar disease has not been analysed.

\section{REFERENCES}

Baker, R., Rosenbaum, A., and Caplan, L. R. (1973). The subclavian steal syndrome. Contemporary Surgery, 4, 96104.

Browne, T. R., III, and Poskanzer, D. C. (1969). Treatment of strokes. New England Journal of Medicine, 281, 594-602, 650-657.

Castaigne, P., Lhermitte, F., Gautier, J. C., Escourolle, R., Derouesné, C., Der Agopian, P., and Popa, C. (1973), Arterial occlusions in the vertebrobasilar system. Brain. 96, 133-154.

Currier, R. D., Giles, C. L., and Westerberg, M. R. (1958). The prognosis of some brain stem vascular syndromes. Neurology (Minneap.), 8, 664-668.

Denny-Brown, D. (1953). Basilar artery syndromes. Bulletin of the New England Medical Center, 15, 53-60.

Fang, H. C. H., and Palmer, J. J. (1956). Vascular phenomena involving brainstem structures. Neurology (Minneap.), 6, 402-419.

Fisher, C. M. (1965a). Lacunes: small, deep cerebral infarcts. Neurology (Minneap.), 15, 774-784.

Fisher, C. M. (1965b). Pure sensory stroke involving face, arm, and leg. Neurology (Minneap.), 15, 76-80.

Fisher, C. M. (1967). A lacunar stroke. The dysarthriaclumsy hand syndrome. Neurology (Minneap.), 17, 614617.

Fisher, C. M. (1969). The arterial lesions underlying lacunes. Acta Neuropathologica (Berl.), 12, 1-15.

Fisher, C. M. (1970). Occlusion of the vertebral arteries. Archives of Neurology, 22, 13-19.

Fisher, C. M., and Caplan, L. R. (1971). Basilar artery branch occlusion: a cause of pontine infarction. Neurology (Minneap.), 21, 900-905.

Fisher, C. M., and Cole, M. (1965). Homolateral ataxia and crural paresis: a vascular syndrome. Journal of Neurology, Neurosurgery, and Psychiatry, 28, 48-55.

Fisher, C. M., and Curry, H. B. (1965). Pure motor hemiplegia of vascular origin. Archives of Neurology, 13, 30-44.

Fisher, C. M., Karnes, W., and Kubik, C. (1961). Lateral medullary infarction, the pattern of vascular occlusion, Journal of Neuropathology and Experimental Neurology, 20, 323-379.

Hill, A. B., Marshall, J., and Shaw, D. A. (1960). A controlled clinical trial of long-term anticoagulant therapy in cerebrovascular disease. Quarterly Journal of Medicine, 29, 597-609.

Jackson, J. H. (1864). Illustrations of diseases of the nervous system. Clinical Lectures and Reports of the London Hospital, 1, 337-387.

Kubik, C. S., and Adams, R. D. (1946). Occlusion of the 
basilar artery - a clinical and pathological study. Brain, 69, 73-121.

Lehrich, J. R., Winkler, G. F., and Ojemann, R. G. (1970). Cerebellar infarction with brain stem compression. Diagnosis and surgical treatment. Archives of Neurology, 22, 490-498.

Marshall, J. (1964). The natural history of transient ischaemic cerebro-vascular attacks. Quarterly Journal of Medicine, 33, 309-324.

Meyer, J. S., Sheehan, S., and Bauer, R. B. (1960). An arteriographic study of cerebrovascular disease in man. 1. Archives of Neurology, 2, 27-45.

Millikan, C. H., Siekert, R. G., and Shick, R. M. (1955). Studies in cerebrovascular disease. 3. The use of anticoagulant drugs in the treatment of insufficiency or thrombosis within the basilar arterial system. Proceedings of the Staff Meetings of the Mayo Clinic, 30, 116-126.

Millikan, C H., Siekert, R. G., and Whisnant, J. P. (1958). Anticoagulant therapy in cerebrovascular disease: current status. Journal of the American Medical Association, 166, 587-592.

North, R. R., Fields, W. S., DeBakey, M. E., and Crawford, E. S. (1962). Brachial-basilar insufficiency syndrome. Neurology (Minneap.), 12, 810-820.

Patel, A., and Toole, J. F. (1965). Subclavian steal syndrome -reversal of cephalic blood flow. Medicine (Balt.), 44, 289-303.

Pochaczevsky, R., Uygar, Z., and Berman, A. J. (1971). Basilar artery occlusion. Journal of the Canadian Association of Radiologists, 22, 261-263.

Pribram, H. F. W. (1965). Complications of cerebral arteriography. In Intracranial Aneurysms and Subarachnoid
Hemorrhage, pp. 184-217. Edited by W. S. Fields and A. L. Sachs. Thomas: Springfield, Ill.

Radner, S. (1951). Vertebral angiography by catheteri zation Acta Radiologica Supplement, 87.

Raymond, F., and Cestan, R. (1901). Trois observations de paralysie des mouvements associés des globes oculaires. Revue Neurologique, 9, 70-77.

Schechter, M. M., and Zingesser, L. H. (1965). The radiology of basilar thrombosis. Radiology, 85, 23-32.

Siekert, R. G., and Millikan, C. H. (1955). Studies in cerebrovascular disease. 2. Some clinical aspects of thrombosis of the basilar artery. Proceedings of the Staff Meetings of the Mayo Clinic, 30, 93-100.

Törmä, T., and Fogelholm, R. (1967). Complications of cerebral angiography with Urografin. Acta Neurologica Scandinavica, 43, 616-629.

Wallenberg, A. (1901). Anatomischer Befund in einem als, "acute Bulbäraffection (Embolie der Art. cerebellar. post. inf. sinistr.?)" Beschriebenen Falle. Archiv für Psychiatrie und Nervenkrankheiten, 34, 923-959.

Weber, H. (1863). A contribution to the pathology of the crura cerebri. Medico-Chirurgical Transactions of the Royal Medical and Chirurgical Society of London, 46, 121-140.

Weibel, J., and Fields, W. S. (1966). Angiography of the posterior cervicocranial circulation. American Journal of Roentgenology, 98, 660-671.

Wheeler, H. B. (1967). Surgical treatment of subclavianartery occlusions. New England Journal of Medicine, 276, 711-717.

Williams, D., and Wilson, T. G. (1962). The diagnosis of the major and minor syndromes of basilar insufficiency. Brain, 85, 741-774.

Williams, D. (1964). Vertebro-basilar ischaemia. British Medical Journal, 1, 84-86. 